\title{
Drug Permeability of Ethylene-Vinyl Alcohol Copolymer Membranes
}

\author{
Shozo Miyazaki; Kuniaki Ishii, and Tanekazu Nadai \\ Faculty of Pharmaceutical Sciences, Josai University, Sakado, Saitama 350-02 (Japan)
}

\begin{abstract}
Synopsis : Ethylene-Vinyl alcohol (EVA) copolymer membrane was evaluated as a new carrier for drug delivery. The steady state permeation of prednisolone and prednisone through EVA copolymer membranes of various comonomer ratios was studied. The rate of drug permeation was found to be dependent upon the monomer composition

(Recieved Mar. 4, 1981)
\end{abstract}

Synthetic polymer membranes have been used as a rate-determing barriers of drug release ${ }^{1)}$. Much of the previous work on controlled-release drug delivery has utilized silicone membranes2).

In the present study, the potential use of ethylenevinyl alcohol (EVA) copolymer as a new carrier for controlled-release drug delivery was investigated. Since EVA copolymer is a heat processing and nontoxic material3), and since the permeability of solute can be varied over a wide range by changes in the monomer composition ${ }^{4}$, exploratory studies on its use as a carrier

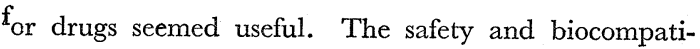
bility of the EVA copolymer are reflected in its use as a hemodialysis membrane3). Therefore, the drug permeability of EVA copolymer membranes was examined first to determine its usefulness as drug delivery devices.

EVA membranes (EVAL) of ethylene contents of $32 \mathrm{~mol} \%$ (Type EF-F) and $47 \mathrm{~mol} \%$ (Type EF-G) were gifts from the Kuraray Co., Tokyo, and the thicknesses of the membranes were claimed to be 15 and $20 \mu \mathrm{m}$, respectively. Two steroidal compounds, prednisolone and prednisone (Merck, Darmstadt), were used as model drugs without further purification. The amount of the drug permeated under a sink condition was determined as described previously5), using plastic dialysis cells of Bahal and Kostenbauder6). A piece of the EVA membrane which had been equilibrated with water was clamped between the two halves of the cell and the assembled cell was placed in an incubator (M-100T, Taiyo Kagaku Kogyo, Tokyo) maintained at $37^{\circ}$. A drug suspension $(20 \mathrm{ml})$ was placed in the donor compartment and the equal volumes of water in the receptor compartment. The cell was shaken horizontally at a rate of 60 strokes/min. The sample solutions were withdrawn periodically, and the solution in the compartment was flushed out and replaced with fresh water. This procedure was used to maintain a sink condition with respect to the permeable species in the receptor solution. Analysis of drugs was carried out spectrophotometrically. All experiments were carried out in triplicate.

The typical permeation profiles of prednisolone and prednisone from suspensions in water through the EVA membranes are shown in Figs. 1 and 2, where the cumulative amount of the drug permeated into the receptor solution is plotted against time. The EVA membranes allowed the steroids to permeate in measurable quantities. When the suspension in water was placed in the donor compartment, the concentration of the steroids in the receptor solution increased linearly with time, since the concentration gradient was virtually constant under the conditions used. An interesting finding is that the rate of permeation of drugs through the EVA membranes depends on the ethylene content in the copolymer. For both the steroids, an increase in the amount of ethy- 
Miyazaki, Ishii, Nadai : Drug Permeability of Ethylene-Vinyl Alcohol Copolymer Membranes

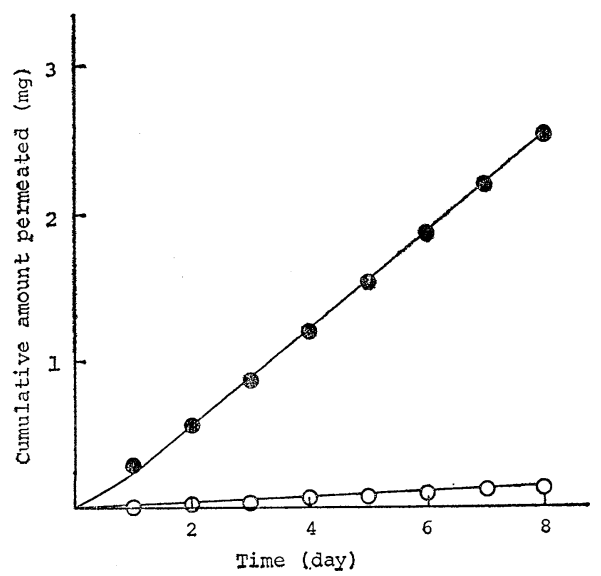

Fig. 1 Permeation profiles of prednisolone through EVA copolymer membranes with $32 \mathrm{~mol} \%$ (2) and $47 \mathrm{~mol} \%$ (O) ethylene contents at $37^{\circ}$

ene content decreases the rate of drug permeation through these polymer composite membranes. The permeability coefficients for these two EVA membranes

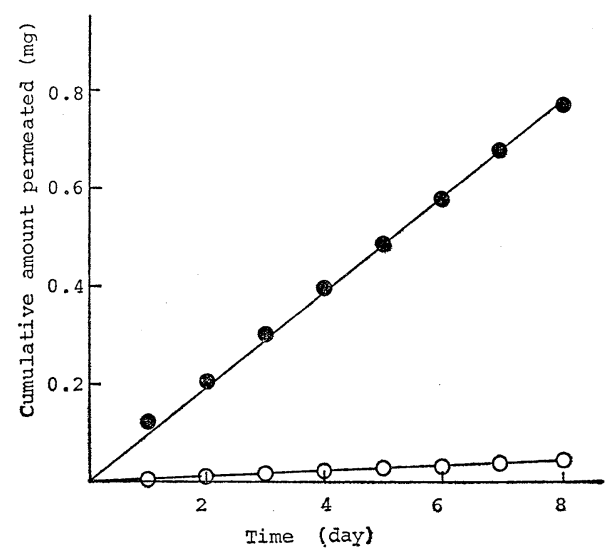

Fig. 2 Permeation profiles of prednisone through EVA copolymer membranes with $32 \mathrm{~mol} \%$ (-) and $47 \mathrm{~mol} \%$ (O) ethylene contents at $37^{\circ}$ with the same comonomers but different comonomer ratios can be estimated from the following simplified steady state treatment7).

$$
Q=\frac{P \cdot A \cdot C \cdot t}{h}
$$

where $Q$ is the amount of drug permeated in time $t$, $C$ is the concentration of the drug in the donor solution, $h$ is the thickness of the membrane, $A$ is the area of the membrane, and $P$ is the permeability coefficient. The average permeability coefficient calculated was $1.3 \times 10^{-9}$ $\mathrm{cm}^{2} \mathrm{sec}^{-1}$ when the copolymer membrane has $32 \mathrm{~mol} \%$ ethylene content and $0.092 \times 10^{-9} \mathrm{~cm}^{2} \mathrm{sec}^{-1}$ when the copolymer membrane has $47 \mathrm{~mol} \%$ ethylene content for prednisolone, and $0.78 \times 10^{-9} \mathrm{~cm}^{2} \mathrm{sec}^{-1}$ and $0.063 \times 10^{-9}$ $\mathrm{cm}^{2} \mathrm{sec}^{-1}$, respectively, for prednisone. Thus it is expected that the release rate may be controlled by modifying the ethylene-vinyl alcohol ratio in the barrier membranes. EVA shows good biocompatibility and should be useful as a unique drug carrier for biomedical implanted, inserted, or surface-applied devices.

\section{References}

1) M. Nakano: Membrane, 3, 386 (1978)

2) J. Folkman, D. M. Long: J. Surg. Res., 4, 139 (1964)

3) T. Hoshino, T. Agishi, Y. Ozaku, I. Kaneko, E. Kumagai, K. Era, K. Ota: Artificial Organs, 7, 264 (1978)

4) H. Iwasaki, K. Hoashi: Kobunshi Ronbunshu, 34, 785 (1977)

5) S. Miyazaki, T. Nadai: Chem. Pharm. Bull., 28, 2261 (1980)

6) C. K. Bahal, H. B. Kostenbauder: J. Pharm. Sci., 53, 1027 (1964)

7) R. E. Lacey, D. R. Cowsar: "Controlled Release of Biologically Active Agents" (A. C. Tanquary, R. E. Lacey, ed.) pp.117-144, Plenum, New York (1974)

\section{Acknowledgement}

Authors are grateful to Kuraray Co. for the supply of ethylene-vinyl alcohol copolymer membranes (EVAL). 